\title{
Insights into metabolic disease from studying genetics in isolated populations: stories from Greece to Greenland
}

\author{
Eleftheria Zeggini $^{1}$ • Anna L. Gloyn ${ }^{2,3,4}$ • Torben Hansen ${ }^{5,6}$
}

Received: 22 January 2016 / Accepted: 25 January 2016 / Published online: 18 March 2016

(C) The Author(s) 2016. This article is published with open access at Springerlink.com

\begin{abstract}
Over the last 10 years substantial progress has been made in our understanding of the genetic basis for type 2 diabetes and related traits. These developments have been facilitated by technological advancements that have allowed comprehensive genome-wide assessments of the impact of common genetic variation on disease risk. Current efforts are now focused on extending this to genetic variants in the rare and low-frequency spectrum by capitalising on nextgeneration sequencing technologies. This review discusses the important contributions that studies in isolated populations are making to this effort for diabetes and metabolic disease, drawing on specific examples from populations in Greece and Greenland. This review summarises a presentation given at the 'Exciting news in genetics of diabetes' symposium at the 2015 annual meeting of the EASD, with topics presented by Eleftheria Zeggini and Torben Hansen, and an overview by the Session Chair, Anna Gloyn.
\end{abstract}

Eleftheria Zeggini

Eleftheria@sanger.ac.uk

1 Wellcome Trust Sanger Institute, Wellcome Trust Genome Campus, Hinxton, Cambridge CB10 1HH, UK

2 Oxford Centre for Diabetes Endocrinology \& Metabolism, University of Oxford, Oxford, UK

3 Wellcome Trust Centre for Human Genetics, University of Oxford, Oxford, UK

4 Oxford NIHR Biomedical Research Centre, Churchill Hospital, Oxford, UK

5 The Novo Nordisk Foundation Center for Basic Metabolic Research, Faculty of Health and Medical Sciences, University of Copenhagen, Copenhagen, Denmark

6 Faculty of Health Sciences, University of Southern Denmark, Odense, Denmark
Keywords Genetic variants · Genome-wide association studies · Isolated populations · Low-frequency variants . Next-generation sequencing technology $\cdot$ Review $\cdot$ Type 2 diabetes

\section{Abbreviation \\ LoF Loss-of-function}

The search for low-frequency and rare variants associated with complex traits requires very large sample sizes to attain the necessary power to detect these effects. Population isolates offer the opportunity to discover such signals with much lower sample sizes. Isolated, or founder, populations have typically gone through a bottleneck event, followed by population expansion with limited rates of migration. These characteristics can offer power advantages in the study of complex trait genetics, especially with respect to the identification of association signals at low frequency, and rare variants that would otherwise be difficult or impossible to detect in cosmopolitan populations $[1,2]$. Some rare variation present in the source population may have been lost in the isolated population, and this can reduce the number of neutral rare variants, which in turn can increase the power of burden tests (i.e. association tests looking for an aggregation of multiple rare variants in a functional unit, e.g. gene or regulatory region). Other rare variants that have made it through the bottleneck can increase in frequency due to drift, or in some cases, selection. This in turn increases the power of association studies as smaller sample sizes are required to detect individual risk loci for complex diseases and traits. In addition, the genomes of isolates have been shaped by population history to typically have longer stretches of linkage disequilibrium. Founder populations also tend to have more homogeneous environmental exposures 
and, in some cases, deep information on genealogy. These attributes can be further enhanced through the ability to recall participants for further characterisation based on genotype and through access to their medical records. Over the last few years, the field of complex trait genetics has witnessed a flourishing of genetic association studies focusing on isolated populations, with the majority of findings replicating across cosmopolitan populations [3-12]. Here, we outline examples from two founder populations stemming from geographical coordinates in Europe ranging from the extreme North and South: Greek individuals from the island of Crete in the south Mediterranean and Inuit individuals from the island of Greenland in the Arctic (Fig. 1).

The inhabitants of the island of Crete in Greece have in the past been investigated through epidemiological studies focusing on dietary and nutrition patterns with respect to metabolic disease [13, 14]. The Hellenic Isolated Cohorts (HELIC) Minoan Isolates (MANOLIS) study was launched in 2008 with the explicit aim of studying the genetic basis of metabolically relevant complex traits in the isolated population residing in the villages of the mountainous Mylopotamos region of Crete (www.helic.org). Anecdotally, this population is known to enjoy good health in old age despite a diet high in animal fat. A total of 1600 recontactable individuals (making up $>10 \%$ of the total population) were recruited into the study and subjected to extensive phenotyping, including anthropometry, detailed diet and lifestyle information, medical history, and blood sample collection for DNA extraction as well as biochemical, haematological and other biomarker analyses. Following genome-wide genotyping and low-depth whole genome sequencing of the entire cohort, association analysis was carried out for quantitative traits of metabolic relevance measured in the population (such as fasting glucose and insulin levels, lipid traits and anthropometry).

Initial population genetic analyses revealed statistically significant enrichment of missense (and therefore potentially functional coding) variants that have substantially risen in frequency in the isolate compared with the general Greek population [15]. A genome-wide scan for association with lipid traits identified a cardioprotective effect at a nonsense variant, p.Arg19Ter, in the $A P O C 3$ gene. The variant abrogates expression of all three isoforms of the gene and has a 40-fold higher frequency in MANOLIS compared with the
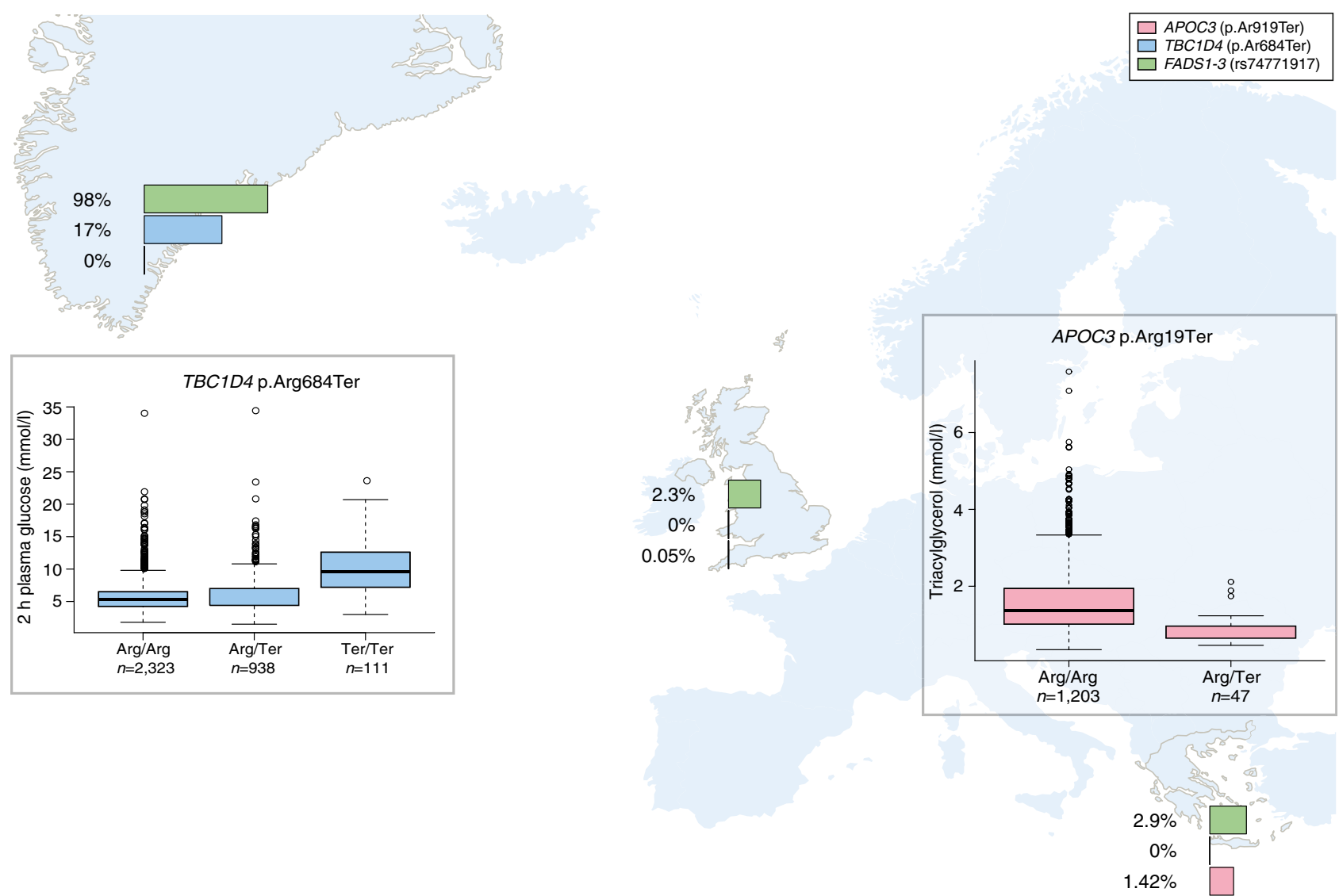

Fig. 1 Allele frequencies (displayed on $\log$ scale) of isolate-enriched variants in Greenland, Crete and the UK general population (UK10K frequencies are taken from www.uk10k.org [21]). Frequency bars are pink for APOC3 p.Arg19Ter (rs76353203) [11], blue for TBC1D4

p.Arg684Ter (rs61736969) [7] and green for FADS2 rs7477191 [18]. The larger graphs show box plots of blood triglyceride levels by APOC3 p.Arg19Ter genotype in Crete, and plasma glucose levels by TBC1D4 p.Arg684Ter in Greenland 
source population (Fig. 1) [11]. The same variant had previously been found to have risen to similar frequency and to have a similar protective association with lipid levels in the Old Order Amish, an independent founder population [8]. Haplotype analysis demonstrated that the variant predates the divergence of both isolates from the general European population. Interestingly, the Amish also have a diet high in animal fat, indicating that the variant may have risen in frequency because of its cardioprotective effect; however, further analyses were not powered to detect selection signatures due to the low allele frequency $(\sim 2 \%)$ [11]. No homozygous individuals were observed in either founder population. Recent work has shown that oligonucleotide-mediated inhibition of APOC3 has therapeutic potential as it decreases blood triacylglycerol (triglyceride) levels [16]. Further recent studies have also identified associations between a burden of multiple loss-of-function (LoF) variants in the $A P O C 3$ gene and lipid levels in the general European population [5, 17]. This story represents a proof-of-principle for the power of isolated populations in medically relevant trait locus discovery, exemplified by an early finding with significant translational potential that is transferable to cosmopolitan populations.

The Greenlandic population is a historically small and isolated founder population. It stands out in at least one important way in comparison with other well-studied founder populations, such as the Finnish and Icelandic populations: these other founder populations are all genetically similar to at least one large population, whereas the Greenlandic Inuit are not closely related to any large population [18]. The Greenlandic Inuit and indigenous populations throughout the Arctic have different fat distribution compared with Europeans [19]. Furthermore, the isolated Greenlandic Inuit population has lower levels of plasma glucose and serum insulin and higher levels of HDL cholesterol than Danes at any given level of obesity [19]. Little is known about how genetics and lifestyle behaviour, particularly physical activity, interact to influence metabolic health in Inuit and whether a recent risk increase of developing type 2 diabetes and related cardiometabolic conditions is driven by a transition from a traditional subsistence lifestyle to a modern, industrialised way of life.

A common Greenlandic Inuit-specific nonsense p.Arg684Ter variant (in which arginine is replaced by a termination codon) in the gene $T B C 1 D 4$, with an allele frequency of $17 \%$, was recently discovered (Fig. 1). It was shown that homozygous carriers of this variant have markedly elevated $2 \mathrm{~h}$ serum insulin levels, postprandial hyperglycaemia, impaired glucose tolerance and a tenfold increased risk of type 2 diabetes [7]. The variant explains about $15 \%$ of type 2 diabetes occurrence in Greenland. Analyses of skeletal muscle biopsies in groups of three individuals carrying zero, one or two copies of p.Arg684Ter revealed that hetero- and homozygous carriers of this stop-gained variant in the gene coding for TBC1D4 are characterised by a marked decrease in the RNA and protein expression of the skeletal muscle-specific long isoform of TBC1D4. Other tissues such as pancreatic beta cells, adipose tissue and liver tissue expressing the short isoform of TBC1D4 were not affected by the stop-gained mutation [7]. Thus, homozygous carriers of p.Arg684Ter could be considered as human muscle-specific knockouts of TBC1D4. Since the variant is common in Greenland (allele frequency of $17 \%$ ) and rare/absent in all other populations, it is possible that selection has historically favoured it in Inuit, possibly because of the low carbohydrate diet associated with traditional Inuit lifestyle. Indeed, weak evidence for positive selection was found [7]. The finding of a common tissue-specific and Inuit-specific LoF variant constitutes proof-of-concept that conducting genetic association studies in small and isolated populations can reveal population-specific type 2 diabetes risk variants and novel biological insights.

While other LoF variants in Inuit may have increased in frequency by chance (genetic drift), it is conceivable that some variants are common specifically in Inuit because of adaptation to the special climate and environment in the Arctic. In fact, a recent study of signs of selection identified genetic variants in fat metabolism in the region of the FADS1-3 genes that alter metabolism of the large amounts of polyunsaturated fatty acids found in the traditional Inuit seafood diet. Gene variants under selection have a strong effect on height and weight, of up to $2 \mathrm{~cm}$ and $4 \mathrm{~kg}$, respectively, as well as a protective effect on cholesterol and triacylglycerol levels [20]. This study illustrates the utility of evolutionary studies of locally adapted populations for understanding the genetic basis of phenotypic variation among humans.

What is becoming increasingly clear is that rare variants of large effect are most likely to be specific to or enriched in particular ancestries. These illustrations from Greece and Greenland provide elegant examples of the opportunities for translational biology offered through the discovery of rare coding alleles that alter disease risk. Detailed physiological studies in carriers of these alleles offer the potential to understand the impact of perturbing particular biological pathways in humans, offering the chance to understand the interplay of proteins in multiple regulatory pathways shedding light on likely adverse on-target effects of therapeutic manipulation.

Acknowledgements The authors thank A. Gilly and I. Tachmazidou (Wellcome Trust Sanger Institute) for generating and contributing Fig. 1, which makes use of data generated by the UK10K Consortium, derived from samples from UK10K COHORTS TWINSUK (The TwinsUK Cohort) and UK10K COHORT ALSPĀC (the Avon Longitudinal Study of Parents and Children). A full list of the investigators who contributed to the generation of the data is available from www.UK10K.org.

Funding EZ is funded by the Wellcome Trust (098051) and the European Research Council (ERC-2011-StG 280559-SEPI). ALG is a Wellcome Trust Senior Fellow in Basic Biomedical Research (095101/ $\mathrm{Z} / 10 / \mathrm{Z}$ ). Funding for UK10K was provided by the Wellcome Trust 
under award WT091310. TH was supported by research grants from The Novo Nordisk Foundation Center for Basic Metabolic Research, an independent research center at the University of Copenhagen partially funded by an unrestricted donation from the Novo Nordisk Foundation.

Duality of interest The authors declare that there is no duality of interest associated with this manuscript.

Contribution statement All authors were responsible for drafting the article and revising it critically for important intellectual content. All authors approved the version to be published.

Open Access This article is distributed under the terms of the Creative Commons Attribution 4.0 International License (http:// creativecommons.org/licenses/by/4.0/), which permits unrestricted use, distribution, and reproduction in any medium, provided you give appropriate credit to the original author(s) and the source, provide a link to the Creative Commons license, and indicate if changes were made.

\section{References}

1. Peltonen L, Palotie A, Lange K (2000) Use of population isolates for mapping complex traits. Nat Rev Genet 1:182-190

2. Zuk O, Schaffner SF, Samocha K et al (2014) Searching for missing heritability: designing rare variant association studies. Proc Natl Acad Sci U S A 111:E455-E464

3. Gudmundsson J, Sulem P, Gudbjartsson DF et al (2012) A study based on whole-genome sequencing yields a rare variant at 8q24 associated with prostate cancer. Nat Genet 44:1326-1329

4. Huyghe JR, Jackson AU, Fogarty MP et al (2013) Exome array analysis identifies new loci and low-frequency variants influencing insulin processing and secretion. Nat Genet 45:197-201

5. Li AH, Morrison AC, Kovar C et al (2015) Analysis of loss-offunction variants and 20 risk factor phenotypes in 8,554 individuals identifies loci influencing chronic disease. Nat Genet 47:640-642

6. Lim ET, Wurtz P, Havulinna AS et al (2014) Distribution and medical impact of loss-of-function variants in the Finnish founder population. PLoS Genet 10:e1004494

7. Moltke I, Grarup N, Jorgensen ME et al (2014) A common Greenlandic TBC1D4 variant confers muscle insulin resistance and type 2 diabetes. Nature 512:190-193
8. Pollin TI, Damcott CM, Shen H et al (2008) A null mutation in human APOC3 confers a favorable plasma lipid profile and apparent cardioprotection. Science 322:1702-1705

9. Sidore C, Busonero F, Maschio A et al (2015) Genome sequencing elucidates Sardinian genetic architecture and augments association analyses for lipid and blood inflammatory markers. Nat Genet 47 : 1272-1281

10. Steinthorsdottir V, Thorleifsson G, Sulem P et al (2014) Identification of low-frequency and rare sequence variants associated with elevated or reduced risk of type 2 diabetes. Nat Genet 46: 294-298

11. Tachmazidou I, Dedoussis G, Southam L et al (2013) A rare functional cardioprotective APOC3 variant has risen in frequency in distinct population isolates. Nat Commun 4:2872

12. Zoledziewska M, Sidore C, Chiang CW et al (2015) Heightreducing variants and selection for short stature in Sardinia. Nat Genet 47:1352-1356

13. Keys A, Menotti A, Karvonen MJ et al (1986) The diet and 15-year death rate in the seven countries study. Am J Epidemiol 124: 903-915

14. Kafatos A, Diacatou A, Labadarios D et al (1993) Nutrition status of the elderly in Anogia, Crete, Greece. J Am Coll Nutr 12:685-692

15. Panoutsopoulou K, Hatzikotoulas K, Xifara DK et al (2014) Genetic characterization of Greek population isolates reveals strong genetic drift at missense and trait-associated variants. Nat Commun 5:5345

16. Graham MJ, Lee RG, Bell TA 3rd et al (2013) Antisense oligonucleotide inhibition of apolipoprotein C-III reduces plasma triglycerides in rodents, nonhuman primates, and humans. Circ Res 112: $1479-1490$

17. TG and HDL Working Group of the Exome Sequencing Project, National Heart, Lung, and Blood Institute (2014) Loss-of-function mutations in APOC3, triglycerides, and coronary disease. N Engl J Med 371:22-31

18. Moltke I, Fumagalli M, Korneliussen TS et al (2015) Uncovering the genetic history of the present-day Greenlandic population. Am J Hum Genet 96:54-69

19. Jorgensen ME, Borch-Johnsen K, Stolk R, Bjerregaard P (2013) Fat distribution and glucose intolerance among Greenland Inuit. Diabetes Care 36:2988-2994

20. Fumagalli M, Moltke I, Grarup N et al (2015) Greenlandic Inuit show genetic signatures of diet and climate adaptation. Science 349: 1343-1347

21. UK10K Consortium, Walter K, Min JL et al (2015) The UK10K project identifies rare variants in health and disease. Nature 526:82-90 